\title{
Entinostat for the treatment of breast cancer
}

Dario Trapani ${ }^{1}$, Angela Esposito ${ }^{1}$, Carmen Criscitiello $^{1}$, Giulia Viale ${ }^{1}$, Luca Mazzarella ${ }^{1}$, Marzia Locatelli $^{1}$, Ida Minchella ${ }^{1}$, Saverio Minucci ${ }^{1,2}$ and Giuseppe Curigliano ${ }^{1}$.

${ }^{1}$ Division of Early Drug Development, European Institute of Oncology, Via Ripamonti 435, Milan, Italy.

${ }^{2}$ University of Milan, Department of Oncology and Hematology, Via Festa del Perdono 7, Milan, Italy.

\section{Corresponding author:}

Giuseppe Curigliano, MD, PhD, Division of Early Drug Development,

European Institute of Oncology, Via Ripamonti 435, 20141 Milan, Italy.

e-mail: giuseppe.curigliano@ieo.it. 


\begin{abstract}
Introduction: Breast cancer accounts for $29 \%$ of malignant tumors. It is an heterogenous disease covering a spectrum of different molecular subtypes. Epigenetic aberrations may affect gene expression through DNA and histone proteins modifications thus promoting tumor progression and resistance to anti- tumor treatment.
\end{abstract}

Area covered: This article explores the potential role of entinostat in the treatment of breast cancer. The clinical trials evaluating entinostat are discussed, highlighting preclinical data and early-phase clinical studies results. The emerging activity of entinostat in several clinical settings is evaluated by focusing on endocrine-resistant, HER2 positive and triple-negative breast cancer with a promising activity as an immune-system boosting.

Expert opinion: Entinostat, a synthetic benzamide derivative class I histone deacetylases (HDACs) inhibitor, inhibits cell proliferation and promotes apoptosis in breast cancer. Several results from clinical trials demonstrate that the addition of an epigenetic therapy to antiestrogen therapy may be an effective approach to targeting resistance pathways in breast cancer, particularly in hormonepositive disease. Agents as entinostat may have a role in immunogenic modulation. Genetic and pharmacological inhibition studies identified HDAC as a key determinant in the reversal of carcinoma immune escape. This offers the rationale for combining HDAC inhibitors with immunotherapy, including therapeutic cancer vaccines.

Keywords: HDAC, endocrine- resistance, entinostat, epigenetic. 


\section{Introduction}

Breast cancer is the mostly diagnosed cancer in women with 249,260 new cases expected for 2016 : it accounts for $29 \%$ malignant tumors in women with a lifelong risk of $12.5 \%$ (1). Breast cancer is an heterogeneous disease covering a spectrum of subtypes with several histological and molecular features affecting prognosis (2). The comprehensive molecular "portrait" of breast cancer reveals four tumor subtypes: luminal A, luminal B, human epidermal growth factor receptor 2 (HER2)overexpressing and basal-like tumors (2), each one characterized by specific genetic and epigenetic abnormalities. Interestingly, these molecular defined subgroups may recapitulate the four main breast cancer intrinsic subtypes as defined in clinical practice through immunohistochemistry assays based on estrogen (ER) and progesterone receptor (PgR), together with HER2- testing and ki-67 as index of proliferation $(3,4)$.

\section{Overview of the epigenetic landscapes of cancer}

The mechanisms of resistance in breast cancer is the result of a clonal evolution. Furthermore, genome instability and intratumor heterogeneity contributes to drug resistance and may promote the selection of resistant subclones, each one with a specific enabling mutant genotype (5). Genomic instability affects the mutational rate of the tumor through several defects: DNA base and nucleotide excision and mismatch repair, telomere maintenance, double-strand break repair, DNA replication, chromosome segregation (6). The differential gene expression can be related to both genetic and epigenetic mechanisms. As a result tumor heterogeneity can be influenced by nonmutational changes affecting gene expression or, properly, by epigenetic mechanisms. These modifications can alter the DNA primary sequence and chromatin compacting regulation through nucleotide or nucleosome-protein modifications. Indeed, each tumor is characterized by a particular epigenetic profile. Neoplastic cells can display different profiles of nucleotidic methylation on cyosine- guanosine ( $\mathrm{CPG}$ ) rich regions (CpG islands): a hypo-methylated profile is associated with chromosomal instability thus impacting on tumor mutagenic rate and outcome (7). Conversely, hyper- methylation of $\mathrm{CpG}$ islands on gene promoter regions leads mostly to gene silencing (8). In addition, expression of tumor suppressor or activating genes can be regulated by post- translational modification of histone proteins such as methylation, acetylation, phosphorylation, ubiquitylation, sumoylation. Histone modifications have been observed in arginine, lysine and serine residues of 
histone proteins (9). Histone-tails modifying enzymes govern these post-translational modifications: histone acetyltransferases (HATs), histone methyltransferases (HMTs), histone deacetylases (HDACs) and histone demethylase (HDMTs). As a general rule, histone acetylation leads to transcriptional activation. Specifically, HDACs are collected in 3 class according to the homology with yeast homologous genes. Class I, in particular, comprises $\operatorname{HDAC} 1,2,3$ and 8, representing the homologous genes to yeast protein RPD3 (10). The acetylation of lysine residues on histone tails neutralizes the positive charge of the $\varepsilon$-amino groups, which is the determinant of the interaction of histone proteins to DNA by binding to the negatively charged phosphodiester backbone, thus influencing the condensation state of DNA. Therefore, a different state of chromatin compaction can allow the access to transcription factors thus enhancing gene expression (Figure 1). Moreover, acetylated lysine can bind reader proteins through acetylated- lysine recognition domain (i.e. bromodomain) thus recruiting diverse nuclear proteins including chromatin remodeling complex proteins and co-activators of transcription. (11). HDACs remove the acetyl group from the histone tail thus enabling the chromatin to condense and restrict access of bromodomain- containing proteins to the DNA, resulting in gene expression negative regulation. Inhibition of HDAC enzymes affect cellular proliferation through the repression of key genes involved in cell-cycle progression, functioning as a cell growth inhibitor. One of the most extensively studied is the cyclin-dependent kinase (CDK) inhibitor CDKN1A / p21 WAF1 (CIP1) that has been showed to be increased in cancer cell lines treated with different HDAC- inhibitors (12). Taken together, the possibility to reverse the epigenetic aberrations that cause chemoresistance and impaired response to targeted therapy of tumor cells is called "episensitization": this possibility of resistance-reversal is attractive as it is amenable to pharmacologic control (13). Interestingly, in-vitro experience with breast cell lines showed an "epigenetic profile" of HDACs expression and mutation. Particularly, an overexpression of HDAC1 has been described in breast and gastric malignant neoplasms (10). This may offer the rationale to target differentially HDAC isoforms as an attempt to target more precisely the different histologically- and molecularly- defined tumors.

\section{Chemistry and pharmacology of entinostat}


2.1 Pharmacodynamics. Entinostat (MS-275) is an oral synthetic benzamide-derivative capable to inhibit HDAC1 and 3 enzymes. Preliminary data showed the potential antiproliferative activity of entinostat in several cell line, including breast cancer in-vitro and in-vivo xenograft models (14). Translational experiences showed that tumor growth suppression requires 3-4 weeks of exposure to entinostat; this is in accordance with the unique mechanism of action of this drug that targets a general thus finely regulated mechanism of gene expression in order to rescue an anti-proliferative expression pattern (15).

2.2 Pharmacokinetics. Pharmacokinetic studies revealed a maximum plasma levels of entinostat reached within $1 \mathrm{~h}$ after a single dose administration and a terminal half-life value estimated between 60 and $150 \mathrm{~h}$ with a t-max equal to $1 \mathrm{~h}(16)$.

2.3 Metabolism. A renal and biliary clearance of entinostat using a radiolabeled model in baboons and cancer patients model has been studied; a possible entero-hepatic recirculation has been supposed (17). However, liver metabolism seems to be a minor pathway of drug elimination in humans, as showed by Ryan et al. (16).

\section{Safety and tolerability}

The safety profile of entinostat is favorable; most frequent grade 3 and 4 adverse events are hematological such as thrombocytopenia (63\%), anemia (47\%), neutropenia (41\%), leukopenia $(10 \%)$ and non- hematological like hypokalemia (8\%), and hypophosphatemia (6\%) unrelated to nephrotoxicity (18).

\section{Preclinical and clinical experiences for breast cancer treatment}

\subsection{Hormone receptor positive breast cancer}

Up to $70 \%$ of breast cancer are ER positive at diagnosis; ER-positive breast cancers at initial diagnosis, however, can lose ER- expression, showing different grades of endocrine therapy resistance (19). ER- $\alpha$ repression is often related to an epigenetic aberration. When treated with entinostat, ER-negative breast cancer cells show an increasing response to endocrine treatment with aromatase inhibitors in a dose dependent manner, so re-sensitizing tumor cells to endocrine therapy (20). Histone modifying enzymes can mediate endocrine-resistance of breast cancer 
through co-repressor proteins. Tamoxifen is an ER modulator capable of inducing a conformational change of ER that favors the recruitment of co-repressors (21). These proteins can interact with HDAC and mediate a trans-repression of ER- related genes. Accordingly, loss of ER-corepressors, due mainly to mutational events or deletion, may affect the response of tumor to endocrinetreatment, altering the compaction of chromatin; again, this same mechanism can predispose cells to the antitumor effect of HDAC- inhibitors (22). Yardley et al. (23) published a phase 2 study (ENCORE 301) of exemestane and entinostat for the treatment of ER-positive breast cancer progressing on treatment with a nonsteroidal aromatase inhibitor. The schedule adopted was entinostat $5 \mathrm{mg}$ PO weekly continued until progressive disease (PD) or unacceptable toxicity. They showed a drug activity for the combination arm in term of progression free survival -PFS ( $H R=0.73, p=0.055)$ and OS (median increase of +8.29 months). Interestingly, ENCORE 301 pharmacodynamics analysis found a predictive role for the hyper-acetylation DNA profile as showed on circulating lymphocytes on liquid biopsy samples: "high acetylators" patients had a better PFS, as found in peripheral blood monocytes, B- and T- lymphocytes with a multiparameter flow cytometry assay (HR=0.32-0.50). The threshold to define the "acetylator" profile was defined as the percent change in protein lysine acetylation at cycle 1 day 15 versus the study derived median value. Thus, a role in endocrineresistance is conceivable with a potential incorporation of entinostat in endocrine- resistant breast cancer treatment in a similar position as CDK4/6 inhibitors, PI3K/mTOR and AKT/PTEN blockers (24). An ongoing phase3 trial (25), sponsored by the national cancer institute ( $\mathrm{NCl})$, will be decisional for the inclusion of entinostat in the armamentarium of this clinical scenario (Table 1).

\subsection{HER2- overexpressing breast cancer}

About $25 \%$ of breast cancer overexpress HER2 oncoprotein and are associated with a worse prognosis than HER2- negative/ER-positive tumors not treated with a targeted therapy (26). At now, anti-HER2 treatment with monoclonal antibodies (i.e. trastuzumab, pertuzumab, TDM-1) and small molecules (lapatinib) are approved in different clinical settings with a significant improvement of the outcome compared to chemotherapy alone. However, resistances to HER2-inhibitors frequently occur, and resistance- crossing strategies are needed (27). The majority of patients under treatment with trastuzumab, despite an initial response, may develop a drug resistance within one year (28). Anti-HER2 treatment resistance can be mediated by the activation of counter-regulatory pathways such as the formation of heterotrimeric complex of HER2 with both HER3 and the insulin-like growth factor-1 (IGF1) or the activation of the PI3K-Akt-mTOR pathway (29). Huang et al reported an 
enhancing activity of entinostat when combined with trastuzumab in HER2-overexpressing breast cancer. Particularly, entinostat exhibited a downregulating effect on HER2 and HER3 with a dramatic inactivation of phosphoinositide 3-kinase (PI3K)/Akt signaling, thus disrupting the two main mechanisms of cancer resistance to trastuzumab treatment (30). Moreover, this activity was confirmed in a trastuzumab- resistant cancer model in which the association of trastuzumab and entinostat exhibited a repressive activity on tumor growth with a substantial enhancement of apoptosis (31). Similarly, Lee J et al (32) reported a synergistic activity of entinostat when combined to lapatinib with a significant in vivo tumor shrinkage. This antitumor activity was showed to be mediated by a downregulation of phosphorylated Akt and activation of the pro-apoptotic protein Bim1. Moreover, when incorporated in a double- blockade regiment with lapatinib and trastuzumab, the antiproliferative effect of entinostat resulted to be improved in comparison with the single anti-HER2 regimen. Based on these results, a phase 1 study is ongoing for trastuzumabresistant HER2-positive breast cancer (33): preliminary results of entinostat and lapatinib with or without trastuzumab showed that the triplet is feasible, safe and effective (34).

\subsection{Triple negative breast cancer}

Triple negative breast cancer subtype (TNBC) encompasses a heterogeneous group of breast malignancies defined by the lack of expression of ER, PgR and HER2. TNBC accounts for almost 15\% of breast malignancies and it is associated with an intrinsic biological aggressiveness and generally with a worse prognosis. At least, six distinct molecular subtypes have been described by Lehmann et al (35), differently associated with outcome and response to neoadjuvant chemotherapy (36). However, no target therapy has been so far approved for TNBC and the treatment of choice remains the cytotoxic chemotherapy. Basal- like subtype of TNBC is characterized by a loss of the expression of $\mathrm{E}$-cadherin and a transition into a mesenchymal- type cell with increasing expression of $\mathrm{N}$ cadherin and vimentin; this process can properly be named "epithelial to mesenchymal transition" (EMT). Specific cell features of EMT are loss of intracellular adhesion molecules, acquisition of spindle fibroblast-like morphology and motility thus acquiring a phenotype suitable for metastatizing (37). However, mutations in $\mathrm{CDH} 1$ gene, encoding for E-cadherin, are rare and the main mechanism of E-cadherin gene regulation seems to be an epigenetic silencing (38). Cells that activate EMT, seems to acquire the features of tumor initiating cells (TICS), characterized by their ability to reseed a tumor even when few cells are inoculated (39). Shah et al (40) showed a reversal of EMT phenotype in a cell model of basal like TNBC exposed to entinostat with a rescue of Ecadherin expression: migration inhibition and cell morphology change was reported together with 
a reduced potential to metastatize. Additionally, entinostat was able to reprogram the TIC phenotype in a basal- like TNBC cellular model: when exposed to entinostat, TNBC cells showed a normalization of $C D 44 / C D 24$ surface expression together with a growth control of the primary tumor and a reduction of the ability of TNBC cancer cells to colonize distant sites (41). Merino et al reported an ex-vivo animal model of combination therapy of entinostat with a differentiating agent (all-trans retinoic acid, ATRA) and doxorubicin (42). In particular, entinostat was able to reverse retinoid acid receptor (RAR- $\beta$ ) epigenetic silencing thus enhancing cell drug sensitivity to ATRA. Additionally, entinostat appeared to reduce topoisomerase II- $\alpha$ and II- $\beta$ expression, defining a synergistic mechanism of action with doxorubicin. According to these preclinical evidences, a phase 1 trial for solid tumors was published by Pili et al (43) with entinostat plus 13-cis retinoic acid; a preliminary evidence of prolonged stable disease was reported in heavenly pretreated patients with prostate, pancreatic and kidney cancer (43).

\subsection{Immune- regulating activity for breast cancer immunotherapy}

The key assumption founding the rational basis for cancer immunotherapy is the concept of immune-surveillance. Immune system, indeed, can suppress the development of cancer clones thus offering an endogenous anti-cancer activity $(44,45)$. The growing success of the "stimulus and costimulus axis" immune-checkpoint regulators have led to clinically validated treatments for a wide spectrum of neoplastic disease; truly, cytotoxic T lymphocyte-associated protein 4 (CTLA-4) and programmed cell death protein 1 (PD1) ligand 1 (PDL-1) blocking monoclonal antibodies have defined a new era of immunotherapy for nearly all types of cancer. When treated with a HDAC inhibitor, breast cancer cells show to be more sensitive to the killing activity of cytotoxic Tlymphocytes (CTL) (46). In particular, HDAC 1 inhibition enhances the antigen- mediated cancer cell killing through a HLA- restricted mechanism. Also, HDAC1 is involved in the epigenetic regulation of genes that participates to the antigen recognition process and co-repressors regulation (47). Moreover, HDAC influences chemokine tumor expression and is capable of interfering with anticancer immune response to immune checkpoint inhibitors like PD-1 blockers, resulting in a brisker T-cell intratumor infiltration and T-cell-dependent tumor regression (48). Furthermore, HDAC inhibition results in an up-regulation of PDL-1, showing that HDAC-inhibitors may synergistically boost anti- PD1/PD-L1 drugs activity (48). In an immune subset analysis from the previously cited ENCORE 301 trial (25), entinostat was confirmed to produce immunomodulatory effects in a cohort of ER- positive breast cancer patients (50). Particularly, an increase of HLA- DR-positive monocytes was observed together with a significant reduction in granulocytic and monocytic myeloid- derived 
suppressor cells. Taken together, these data show an immune-stimulating switch of the association therapy with exemestane and entinostat, hence providing a rationale for immune- checkpoint inhibitor association to endocrine therapy and HDAC- modulators (Table2).

\section{Competitor drugs in development}

Clinical drug development of entinostat is focusing on breast cancer resistance mechanisms to endocrine therapy and to HER2-targeting agents. Therefore, clinical competitors are being developing contemporary in diverse clinical settings. Endocrine resistance in breast cancer involves different pathways of interest: ER-alpha expression levels, up-regulation of growth factors signaling pathways (HER2, IGFR1, and FGFR1), downstream activation of the mitogen-activated protein kinase (MAPK) or PI3K cascade, CDK4/6, and let-7 miRNA family dysregulation (51). Similarly, trastuzumab resistance can be determined by several aberrations that interest PI3K/ mTOR pathway, cell- cycle control, IGFR1, MAPK (52). For at least each of these anomalies, a drug is under clinical evaluation and some of them are reaching a definite position in clinical field within few months.

\section{Expert opinion.}

Entinostat is a promising drug under development for the treatment of breast cancer in different clinical settings. Its unique mechanism of action interferes with a general mechanism of tumorigenesis, involving several and different oncogenic pathways that promote and sustain tumor progression. Conversely, entinostat targets class I HDACS (HDAC 1 and 3) so defining a tailored mechanism of action with a specific antitumor activity, too. In particular, entinostat can be combined with targeted- therapy as a booster of clinical activity as well as in association with immune-targeting treatments in support of a stronger antitumor immunity response and incorporated in chemotherapy regimens with potential synergistic activity. In addition, entinostat has a long half- life and can be administered once weekly PO, making this medication unique compared with other HDAC "broad spectrum" modulators such as azacitidine. Even though long term side effects are not completely known, Entinostat may be used for long term and beyond progression of disease -even if contrasting data exists (58)- expecting a persistent antitumor effect: this class of epigenetic- modulator drugs may create a favorable genomic profile prone to a proapoptotic and immune- activating context that may act in a promiscuous manner with several combined and sequential partner-drugs. Furthermore, patients may be monitoring before, during and at progression with liquid biopsy to detect a peculiar epigenetic profile predictive of response 
to entinostat on circulating cancer cells and immune- competent cells or identify emerging mutation or epigenetic aberration of resistance to HDAC modifiers. Moreover, some of this epigenetic changes (i.e. cycle 1 day 15 lysine acetylation change) may be an early predictor of response to entinostat. However, further prospective data on endocrine resistance reversal, long term toxicity and immune- related adverse events enhancing are still needed. In an optimistic scenario, entinostat will be a promiscuous and transversal drug to be prescribed for almost all breast cancer subtypes as well as in several clinical setting as a general booster of antitumor activity.

Table 1. Synoptic table of entinostat

\begin{tabular}{|l|l|}
\hline Drug name (company name) & $\begin{array}{l}\text { Entinostat (Syndax Pharmaceuticals, Inc., } \\
\text { Waltham, MA) } \\
\text { Other names: SNDX-275, MS-275 }\end{array}$ \\
\hline
\end{tabular}




\begin{tabular}{|ll|}
\hline Phase & 1,2 and 3, ongoing \\
Indication & Hodgkin's lymphoma, leukemia, lung cancer, \\
Route of administration & breast cancer, melanoma, ovarian cancer \\
Chemical structure & Oral \\
& $\mathrm{C}_{21} \mathrm{H}_{20} \mathrm{~N}_{4} \mathrm{O}_{3}$ \\
& Pyridylmethyl- $\mathrm{N}-\{4-[(2$-aminophenyl)- \\
& carbamoyl]-benzyl\}-carbamate \\
Pivotal trials ongoing: Ref & $22,24,32,33,52,53,54,55,56$ \\
\hline
\end{tabular}

Table 2. Ongoing trials with entinostat for the treatment of breast cancer

\begin{tabular}{|c|c|c|c|c|}
\hline Partner- Drug & Phase & Disease & Setting & $\begin{array}{l}\text { ClinicalTrials.gov } \\
\text { identifier }\end{array}$ \\
\hline $\begin{array}{l}\text { Exemestane } \\
\text { Goserelin }\end{array}$ & III & HRBC & $\mathrm{LABC} / \mathrm{M}$ & NCT02115282 \\
\hline Anastrozole & II & TNBC & Neoadjuvant & NCT01234532 \\
\hline $\begin{array}{l}\text { Nivolumab } \\
\text { Ipilimumab }\end{array}$ & 1 & $\begin{array}{l}\text { HER2- } \\
\text { negative Breast Cancer }\end{array}$ & $\mathrm{LABC} / \mathrm{M}$ & NCT02453620 \\
\hline Azacitidine & II & HRBC, TNBC, HER2-positive & $\mathrm{LABC} / \mathrm{M}$ & NCT01349959 \\
\hline Exemestane & 1 & HRBC & $\mathrm{LABC} / \mathrm{M}^{*}$ & NCT02833155 \\
\hline Atezolizumab & $\mathrm{Ib}$ & TNBC & $\mathrm{LABC} / \mathrm{M}$ & NCT02708680 \\
\hline Pembrolizumab & 1 & HRBC, TNBC, HER2-positive & $\mathrm{LABC} / \mathrm{M}$ & NCT02909452 \\
\hline $\begin{array}{l}\text { Trastuzumab } \\
\text { Lapatinib }\end{array}$ & 1 & HER2- positive & $\mathrm{LABC} / \mathrm{M}$ & NCT01434303 \\
\hline
\end{tabular}

$B C$, breast cancer. $H R B C$, estrogen and/or progesterone receptor-positive $B C$. TNBC, triple negative $B C$. HER2, human epidermal growth factor receptor 2. $L A B C / M$, locally advanced or metastatic breast cancer (palliative setting). *Only Chinese postmenopausal patients (Last update October 2016). 
Figure 1. Epigenetics modifications are involved in endocrine-resistance of breast cancer 


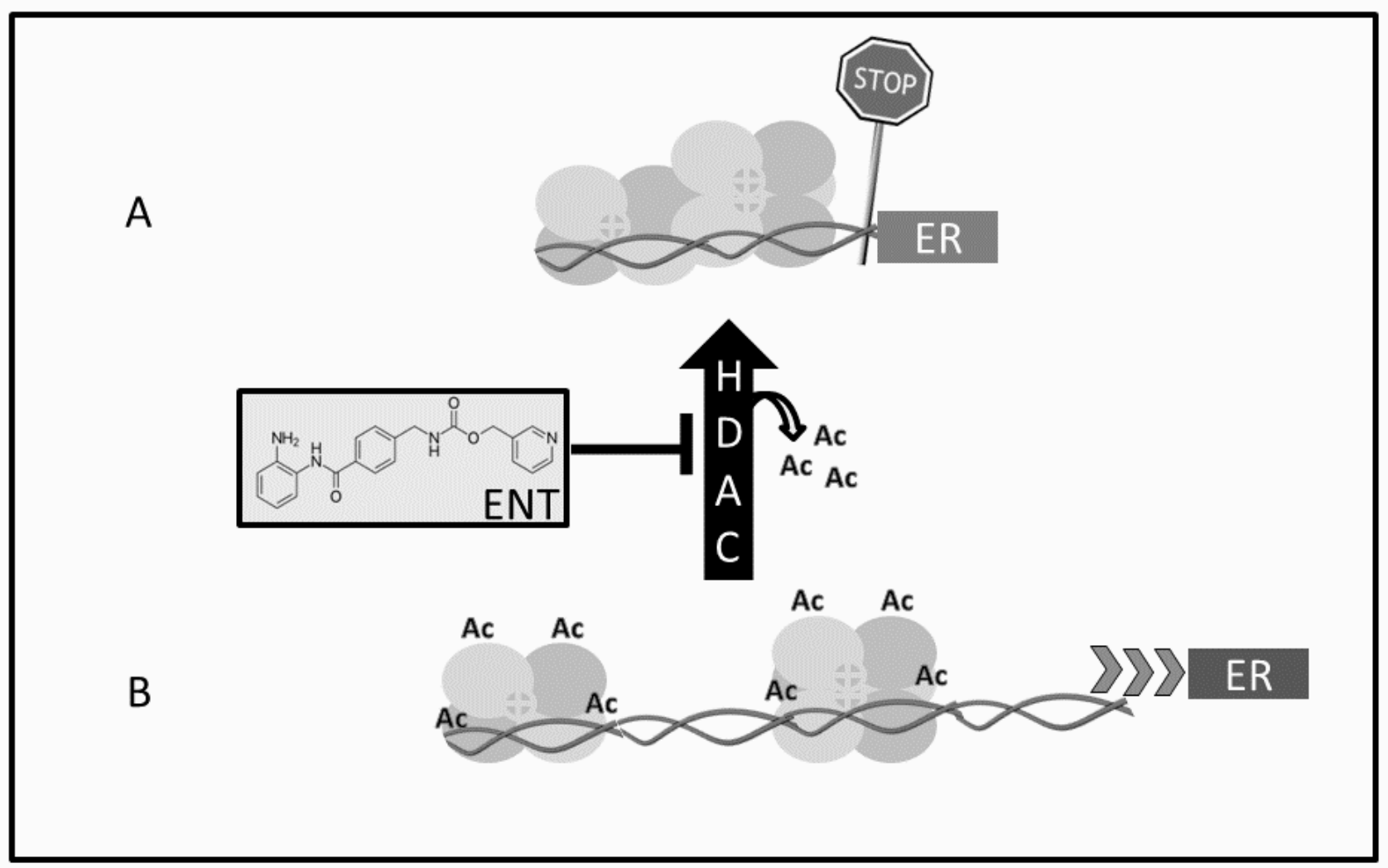

The acetylation of lysine residues on histone tails determines a different state of chromatin condensation thus allowing access to transcription factors and possibly, but not only, enhancing gene expression. Aberrations in the acetylation pattern of cancer cells DNA can repress the expression of estrogen receptor $(A)$ as one of mechanism leading to antitumor activity. When treated with entinostat (B), endocrine- resistant breast cancer cells show an increasing response to the treatment with exemestane, displaying a re-sensitization to aromatase inhibitors. Similar regulatory mechanisms of gene expression are involved in the re- sensitization to trastuzumab in breast cancer progressing to anti-HER2 treatment as well as in the immune- modulatory effects of entinostat (see the text for more details). HDAC, Histone deacetylase 1 and 3. ENT, Entinostat. ER, oestrogen receptor.

\section{References}


1- CA Cancer statistics, 2016. Cancer J Clin 2016;7-30.

2- The Cancer Genome Atlas Network. Comprehensive molecular portraits of human breast tumours. Nature 2012; 490: 61-70.

3- Coates AS, Winer EP, Goldhirsch A et al. Tailoring therapies-improving the management of early breast cancer: St Gallen International Expert Consensus on the Primary Therapy of Early Breast Cancer 2015. Ann Oncol 2015; 26:1533-1546.

4- Senkus E, Kyriakides E, Ohno S, Penault-Llorca F et al. Primary breast cancer: ESMO Clinical Practice Guidelines for diagnosis, treatment and follow-up. Annals of Oncology 26: v8-v30, 2015.

5- Venkatesan S. Tumor Evolutionary Principles: How Intratumor Heterogeneity Influences Cancer Treatment and Outcome 2016; 35: e141-9.

6- Burrel AR, McGranah N, Bartek J, Swanton C. The causes and consequences of genetic heterogeneity in cancer evolution, Nature 2013;Volume: 501,Pages:338-345. **

**this is a military stone in cancer evolution comprehension and mechanisms of resistance to targeted- therapies identification

7- Goelz SE, Vogelstein B, Hamilton SR, Feinberg AP. Hypomethylation of DNA from benign and malignant human colon neoplasms. Science 228, 187-190.

8- Esteller M, Corn PG, Baylin SB, Herman JG. A gene hypermethylation profile of human cancer. Cancer Res. 61, 3225-3229.

9- Esteller M. Cancer epigenomics: DNA methylomes and histone-modification maps. Nat Rev Genet 2007; 8:286-298.

10- Ozda GH, Teschendorff AE, Ahmed AA, Hyland SJ et al. Differential expression of selected histone modifier genes in human solid cancers. BMC Genomics 7, 90. *

*this is a general article focusing on epigenetic alterations and hypothetical mechanism of tumorigenesis; furthermore, it represents the most comprehensive analysis of the expression alterations of epigenetic- changes involved genes in human cancers and their corresponding normal tissues 
11- Verdin E, Ott M. 50 years of protein acetylation: from gene regulation to epigenetics, metabolism and beyond. Nature Reviews Molecular Cell Biology: 16, 258-264.

12- Hess-Stumpp H. Histone deacetylase inhibitors and cancer: from cell biology to the clinic. Eur J Cell Biol 2005; 84: 109-21.

13- Oronsky B, Oronsky N, Knox S, Fanger G et al. Episensitization: Therapeutic Tumor Resensitization by Epigenetic Agents: A Review and Reassessment . Anti-Cancer Agents in Medicinal Chemistry, 2014; 1121-1127.

14- Saito A, Yamashita T, Mariko Y, et al. A synthetic inhibitor of histone deacetylase, MS-27275, with marked in vivo antitumor activity against human tumors. Proc Natl Acad Sci U S A 1999; 96:4592-7.

15-Sausville EA, Alley MC, Pacula-Cox CM et al. Pharmacologic evaluations of MS-275 (NSC706995), a novel benzamide structure with a unique spectrum of antitumor activity. Proc Am Assoc Cancer Res 2001;42:927.

16- Ryan QC, Headlee D, Acharya M, Sparreboom A et al. Phase I and pharmacokinetic study of MS-275, a histone deacetylase inhibitor, in patients with advanced and refractory solid tumors or lymphoma. J Clin Oncol. 2005 Jun 10;23:3912-22.*

*this is one of the first experience published in literature with MS-275 as a regulator of epigenetic regulations in solid tumors

17- Hooker JM, Kim SW, Alexoff D, Xu Y et al. Histone deacetylase inhibitor, MS-275, exhibits poor brain penetration: PK studies of [C]MS-275 using Positron Emission Tomography. ACS Chem Neurosci. 2010:65-73.

18- Batlevi CL, Kasamon Y, Bociek RG, Lee P et al. ENGAGE- 501: phase II study of entinostat (SNDX-275) in relapsed and refractory Hodgkin lymphoma. Haematologica. 2016 Aug;101:968-75.

19- Yang X, Phillips DL, Ferguson AT et al. Synergistic activation of functional estrogen receptor (ER)-alpha by DNA methyltransferase and histone deacetylase inhibition in human ER-alphanegative breast cancer cells. Cancer Res 2001;61:7025-7029. **

** this article explores the role of epigenetic methylation on tumorigenesis of ER-negative breast cancer (i.e. triple-negative) 
20-Sabnis JG, Goloubeva O, Chumsri S, Nguyen N et al. Functional activation of the estrogen receptor- $\alpha$ and aromatase by the HDAC inhibitor, entinostat, sensitizes of ER-negative tumors to letrozole. Cancer Res. 2011 March 1; 71: 1893-1903. *

*this paper defines a new role of ER- signaling in ER-negative breast tumor by a resensitization targetable through an epigenetic modulation

21- Heldring N, Nilsson M, Buehrer B, Treuter E et al. Identification of tamoxifen-induced coregulator interaction surfaces within the ligand-binding domain of estrogen receptors. Mol Cell Biol. 2004: 3445-3459.

22- Légaré S, Basik M. Minireview: The Link Between ER $\alpha$ Corepressors and Histone Deacetylases in Tamoxifen Resistance in Breast Cancer. Mol Endocrinol. 2016 Sep;30:965-76.

23-Yardley DA, Ismail-Khan RR, Melichar B, Lichinitser M et al. Randomized Phase II, DoubleBlind, Placebo-Controlled Study of Exemestane With or Without Entinostat in Postmenopausal Women With Locally Recurrent or Metastatic Estrogen Receptor-Positive Breast Cancer Progressing on Treatment With a Nonsteroidal Aromatase Inhibitor. J Clin Oncol. 2013 Jun 10; 31:2128-35.

24- Perez EA. Treatment strategies for advanced hormone receptor-positive and human epidermal growth factor 2-negative breast cancer: the role of treatment order. Drug Resistance 2016: 13-22

25- NCT02115282: https://clinicaltrials.gov/ct2/show/NCT02115282

26- Ross JS, Slodkowska EA, Symmans WF, Pusztai L et al. The HER-2 receptor and breast cancer: ten years of targeted anti-HER-2 therapy and personalized medicine. Oncologist 2009; 14: 320-68.

27-Slamon DJ, Leyland-Jones B, Shak S, et al. Use of chemotherapy plus a monoclonal antibody against HER2 for metastatic breast cancer that overexpresses HER2. N Engl J Med 2001; 344: 783-92.

28- Valabrega G, Montemurro F, Aglietta M. Trastuzumab: mechanism of action, resistance and future perspectives in HER2-overexpressing breast cancer, Ann. Oncol. 18 (2007) 977-984.

29- Wang L, Zhang Q, Zhang J, Sun S et al. PI3K pathway mactivation results in low efficacy of both trastuzumab and lapatinib. BMC Cancer. 2011;11: 248. 
30- Huang X, Gao L, Wang S, Lee CK et al. HDAC inhibitor SNDX-275 induces apoptosis in erbB2overexpressing breast cancer cells via down-regulation of erbB3 expression, Cancer Res. 2009: 8403-8411.

31- Huang $X$, Wang S, Lee CK, Yang $X$ et al. HDAC inhibitor SNDX-275 enhances efficacy of trastuzumab in erbB2-overexpressing breast cancer cells and exhibits potential to overcome trastuzumab resistance. Cancer Lett. 2011 Aug 1;307:72-9.

32-Lee J, Bartholomeusz C, Mansour O, Humphries J et al. A class I histone deacetylase inhibitor, entinostat, enhances lapatinib efficacy in HER2overexpressing breastcancer cells through FOXO3-mediated Bim1 expression. Breast Cancer ResTreat. 2014; 146: 259-72.

33- NCT01434303: https://clinicaltrials.gov/ct2/show/NCT01434303.

34- Lim B, Murthy RK, Jackson S, Willey JS et al. Open-label phase Ib study of entinostat (E), and lapatinib (L) alone, and in combination with trastuzumab (T) in patients (pts) with HER2+ metastatic (mHER2+) breast cancer after progression on trastuzumab. J Clin Oncol 2016: 609.

35- Lehmann BD, Bauer JA, Chen X, Sanders ME et al. Identification of human triple-negative breast cancer subtypes and preclinical models for selection of targeted therapies. J Clin Invest. $2011 \mathrm{Jul} ; 121: 2750-67$.

36- Masuda H, Baggerly K, Wang $Y$, Zhang $Y$ et al. Differential response to neoadjuvant chemotherapy among 7 triple-negative breast cancer molecular subtypes. Clin Cancer Res: 5533-5540.

37- Blick T, Widodo $E$, Hugo $H$, Waltham $M$, et al. Epithelial mesenchymal transition traits in human breast cancer cell lines. Clin Exp Metastasis, 2008. 25:629-642.

38-Zou D, Yoon HS, Perez D, Weeks RJ et al. Epigenetic silencing in non-neoplastic epithelia identifies E-cadherin (CDH1) as a target for chemoprevention of lobular neoplasia 2009. J Pathol 218:265-272.

39- Reya T, Morrison SJ, Clarke MF, Weissman IL. Stem cells, cancer, and cancer stem cells. Nature 2001;414:105-11.

40-Shah P, Gau Y, Sabnis G. Histone deacetylase inhibitor entinostat reverses epithelial to mesenchymal transition of breast cancer cells by reversing the repression of $E$ cadherinBreast Cancer Res Treat 2014. 143:99-111.

41- Schech A, Kazi A, Yu S, Sabnis G. Histone Deacetylase Inhibitor Entinostat Inhibits TumorInitiating Cells in Triple-Negative Breast Cancer Cells. Mol Cancer Ther. 2015. 14:1848-57. 
42- Merino

VF, Nguyen

$\mathrm{N}, \mathrm{Jin}$

K, Sadik

$\mathrm{H}$,

et

al.

Combined Treatment with Epigenetic, Differentiating,and Chemotherapeutic Agents Coope ratively TargetsTumor-Initiating Cells in Triple-Negative Breast Cancer. Cancer Res. 2016 Apr 1;76:2013-24.

43- Pili R, Salumbides B, Zhao M, Altiok S et al. Phase I study of the histone deacetylase inhibitor entinostat in combination with 13-cis retinoic acid in patients with solid tumours. Br J Cancer 2012. 106: 77-84.

44- Nanda R, Chow LQ, Dees EC, Berger R et al. Pembrolizumab in Patients With Advanced Triple-Negative Breast Cancer: Phase Ib KEYNOTE-012 Study. J Clin Oncol. 2016. 34(21):2460-7.

45-Shankaran V, et al. IFNy and lymphocytes prevent primary tumor development and shape tumor immunogenicity. Nature. 2001;410:1107-1111.

46- Gameiro SR, Malamas AS, Tsang KY, Ferrone S et al. Inhibitors of histone deacetylase 1 reverse the immune evasion phenotype to enhance T-cell mediated lysis of prostate and breast carcinoma cells. Oncotarget. 2016. 7390-402.

47-Yang PM, Lin PJ, Chen CC. CD1d induction in solid tumor cells by histone deacetylase inhibitors through inhibition of HDAC1/2 and activation of Sp1. Epigenetics. 2012. 390-9.

48-Zheng $H$, Zhao $\quad W$, Yan $\quad$, Watson $\quad C C$ et HDAC Inhibitors Enhance TCell Chemokine Expression and Augment Response to PD1 Immu notherapy inLung Adenocarcinoma. Clin Cancer Res. 2016. 22:4119-32
49- Woods
DM, Sodré
$\mathrm{AL}$, Villagra
A, Sarnaik
A
et
al.

HDAC Inhibition Upregulates PD1 Ligands in Melanoma and Augments Immunotherapy with PD-1 Blockade. Cancer Immunol Res. 2015. 3:1375-85.

50- Tomita Y, Lee MJ, Lee S, Tomita S et al. The interplay of epigenetic therapy and immunity in locally recurrent or metastatic estrogen receptor-positive breast cancer: Correlative analysis of ENCORE 301, a randomized, placebo-controlled phase II trial of exemestane with or without entinostat. Oncolmmunology 2016. *

* this article shows an interesting analysis of the results of a phase- 3 trial with entinostat in breast cancer patients with IO- observations

51- Haynes E, Lewis- Wambi JS. Mechanisms of endocrine resistance in breast cancer: an overview of the proposed roles of noncoding RNA Breast Cancer Research 2015. 17:40. 
52-Luque-Cabal $\quad M$, García-Teijido $\quad P$, Fernández-Pérez $\quad Y$, Sánchez-Lorenzo $\quad L \quad$ et $\quad$ al. Mechanisms Behind the Resistance to Trastuzumab in HER2Amplified Breast Cancer and St rategies to Overcome It. Clin Med Insights Oncol. 2016: 10:21-30.

53- NCT00387465: https://clinicaltrials.gov/ct2/show/NCT00387465?term=entinostat\&rank=5 54- NCT02915523: https://clinicaltrials.gov/ct2/show/NCT02915523?term=entinostat\&rank=2 55- NCT00866333:

https://clinicaltrials.gov/ct2/show/NCT00866333?term=entinostat\&rank=31

56- NCT00313586:

https://clinicaltrials.gov/ct2/show/NCT00313586?term=entinostat\&rank=25

57- NCT02437136:

https://clinicaltrials.gov/ct2/show/NCT02437136?term=entinostat\&rank=16

58- Connolly R, Li H, Jankowitz RC, Zhang Z et al. Combination Epigenetic Therapy in Advanced Breast Cancer with 5-Azacitidine and Entinostat: A Phase II National Cancer Institute/Stand Up to Cancer Study. Clin Cancer Res.2016. 